\title{
Estimación del costo de distribución de la energía eléctrica en Colombia considerando generación distribuida fotovoltaica
}

\author{
Carlos A. García Montoya ${ }^{1,2}$, Jesús M. López-Lezama ${ }^{3}$ y Tomás Gómez San Román ${ }^{4}$ \\ (1) Facultad de Minas, Universidad Nacional de Colombia, Carrera 80 No 65-223, Medellín, Colombia \\ (correo-e: cgarciamo@unal.edu.co) \\ (2) Empresas Públicas de Medellín, Carrera 58 No 42-125, Medellín, Colombia \\ (correo-e: carlos.garcia.montoya@epm.com.co) \\ (3) Departamento de Ingeniería Eléctrica, Universidad de Antioquia, Calle 67 No 53-108, Medellín, Colombia \\ (correo-e: jmaria.lopez@udea.edu.co) \\ (4) Instituto de Investigación Tecnológica, Universidad Pontificia Comillas, Madrid, España \\ (correo-e: tomas.gomez@comillas.edu)
}

Recibido Jul. 1, 2020; Aceptado Ago. 26, 2020; Versión final Nov. 16, 2020, Publicado Feb. 2021

\begin{abstract}
Resumen
Este estudio presenta una evaluación del impacto de la penetración de generación distribuida fotovoltaica (GDF) en los costos de distribución de electricidad, proporcionando a las empresas de distribución una herramienta para estimar el impacto de la penetración de GDF a pequeña escala. Para estimar tal impacto, se utilizan modelos de red de referencia con el objetivo de estimar las variaciones de costos y el rendimiento de la infraestructura, considerando los cambios tecnológicos debido a la integración de GDF en el sistema de distribución. El estudio se ilustra en un sistema real con 1000 transformadores de distribución y 11000 clientes. Se obtuvo que principalmente los valores de costos de inversión de la infraestructura están estimados en 39033 Millones de pesos colombianos (MCOP) y los costos de mantenimiento en 3020 MCOP. Adicionalmente, no se observan mayores cambios en la longitud de la red. Se conluye que la GDF no presenta una alta incidencia dentro de los costos de la red de distribución.
\end{abstract}

Palabras clave: costos; distribución; generación distribuida fotovoltaica; modelos; red; referencia

\section{Cost estimation of Colombian electric power distribution by considering distributed photovoltaic generation}

\begin{abstract}
This study presents a distributed photovoltaic generation (DPG) impact assessment for electric power distribution costs in Colombia. It also provides a tool for energy distribution companies to estimate the impact of DPG small scale penetration. Reference network models are used to estimate cost variations and infrastructure performance by considering technological changes caused by DPG integration in the distribution system. A real system with 1,000 distribution transformers and 11,000 customers is used. The results show that the infrastructure investment cost estimate is 39,033 million Colombian pesos (MCOP) and that the maintenance cost estimate is 3,020 MCOP. It is concluded that DPG does not have a major impact on distribution network costs.
\end{abstract}

Keywords: distribution network costs; distributed photovoltaic generation; reference network models 


\section{INTRODUCCIÓN}

En las últimas décadas varios países han cambiado la composición de su matriz energética migrando hacia la integración de energías renovables no convencionales. Avances tecnológicos en la generación de electricidad a pequeña escala, crecientes restricciones ambientales para la construcción de grandes generadores, la preocupación por la dependencia de combustibles fósiles y el fenómeno del calentamiento global han sido los principales impulsores de esta transformación (Huang et al., 2019). Dentro de las tecnologías de generación basadas en fuentes renovables no convencionales con mayor auge se destacan la eólica y solar fotovoltaica. Esta última desarrollándose principalmente bajo el esquema de generación distribuida. La generación fotovoltaica exhibe ventajas frente a otro tipo de tecnologías debido especialmente a su fácil instalación y a la notable reducción de sus costos debido a recientes desarrollos tecnológicos (Branker et al., 2011). De hecho, se han llegado a instalar grandes campos de generación fotovoltaica permitiendo conectarse directamente a los sistemas de transmisión o distribución de energía eléctrica (Moore y Post, 2008). En contraste, también se han adoptado estrategias de desarrollo basadas en pequeños centros de generación distribuidos y conectados al sistema de distribución, desde el lado del consumidor, denominada GDF (Toledo et al., 2010).

La masificación de la GDF y de la generación distribuida en general ofrece ventajas de lado del consumidor, como también nuevos desafíos para el regulador del mercado y el operador de la red de distribución. Uno de los desafíos más notables es el efecto que tiene este tipo de tecnologías sobre la operación del sistema de distribución y en los costos de distribución de energía (Eltawil y Zhao, 2010). Desde la perspectiva del regulador, la instalación de GDF demanda el desarrollo de nuevos mecanismos que permitan e incentiven la conexión de energías limpias y de parte del distribuidor se requiere conocer cuál es el impacto sobre los costos de la empresa de distribución, así como el efecto de los nuevos equipos instalados sobre la infraestructura existente. La evaluación de estos elementos es de vital importancia de cara a garantizar la sostenibilidad del negocio y su adaptabilidad. Colombia no ha sido ajena a esta realidad, y en la actualidad, el gobierno nacional, por medio del Ministerio de Minas y Energía y de la UPME (Unidad de Planeación Minero Energética), está desarrollando un marco regulatorio que incentive el desarrollo de fuentes de energía alternativa no convencionales (Gallego et al., 2017; Restrepo et al., 2016).

La penetración de la GD en los sistemas de distribución ha llevado a la necesidad de desarrollar diferentes estudios en busca de identificar y estimar la magnitud de sus efectos. En términos generales, la GD puede tener impactos positivos como la reducción de pérdidas de potencia, el mejoramiento del perfil de tensiones, el aplazamiento de inversiones o refuerzos en la red y la reducción de emisiones de efecto invernadero. Sin embargo, su inadecuado planeamiento puede traer inconvenientes como el aumento de pérdidas o problemas en la coordinación de protecciones (Zhan et al., 2016). Es por esto que muchos autores han desarrollado metodologías para la correcta ubicación y dimensionamiento de GD. Estas metodologías se pueden clasificar en dos grandes grupos: técnicas metaheurísticas y optimización matemática clásica. Estas últimas pueden garantizar la optimialidad de la solución encontrada, pero requieren linealizaciones y simplificaciones para ser aplicadas con efectividad. Por otro lado, dada la naturaleza no lineal y no convexa del problema de ubicación y dimensionamiento óptimo de GD, las técncias metaheurísticas se han ajustado más a este desafío.

Las técnicas de optimización metaheurística se basan normalmente en analogías de procesos físicos o biológicos, así como en el comportamiento de algunos grupos de animales. En (Vasileios y Pavlos, 2014) se presenta un algoritmo genético para la ubiación y dimensionamiento óptimo de GD considerando incertezas. Para ello los autores implementan un flujo de potencia probabilistio que considera la incerteza en la demanda y en las fuentes de generación. En (Hassan et al. 2020) los autores presentan un algoritmo basado en enjambre de partículas para optimizar la ubicación y dimensionamiento de GD renovable en sistemas de distribución. En este caso, los autores consideran generación eólica y solar fotovoltaica. En (Wang et al., 2018) se presenta una metodología para el dimensionamiento óptimo de GD en microrredes considerando diferentes modos de operación considerando DG eólica y fotovoltaica, además de un sistema de almacenamiento. En (Pereira et al., 2016) se propone una metaheuristica híbrida que combina un algoritmo genético con una búsqueda tabú para determinar de forma simultánea la ubicación y dimensionamiento óptimo de GD y bancos de capacitores en sitemas de distribución. La metodología propuesta también tiene en cuenta la naturaleza estocástica de las fuentes de generación. Una revisión bibliográfica sobre diferentes métodos aplicados a la ubicación y dimensionamiento óptimo de GD en sistemas de distribución puede ser consultada en (Adefarati y Bansal, 2016).

Dentro de los aspectos de interés en los estudios de la penetración de la GD se encuentran los costos en los que debe incurrir la empresa de distribución al momento de enfrentar el ingreso de la GD en el sistema y los estudios de viabilidad técnico-económica de las inversiones que deben realizarse para adecuar el sistema a las nuevas condiciones (Zou et al., 2017). Algunos estudios se han centrado en el enfoque de estimación de la viabilidad económica fundamentada en análisis de costo beneficio (Yong, et al., 2019), costos de fabricación (Paul, 2014), costos técnicos de operación (Normandia et al., 2019) y costos de diseño (Kerekes et al., 2013). 
Sin embargo, pocos trabajos se han enfocado en el costo de la distribución desde el enfoque de las empresas de distribución.

En el presente artículo se evalúa el impacto de la penetración de la GDF sobre costos de la distribución de energía eléctrica en un caso de estudio en Colombia. Esto con el objetivo de proveer a las empresas de distribución con valores estimados de los costos, de modo tal que se pueda prever su efecto, especialmente, sobre el desempeño de la empresa y su sostenibilidad frente a los cambios regulatorios. Para lograr este objetivo se evalúa el impacto de la GDF en un sistema de distribución mediante el uso de un modelo de red de referencia (RNM por sus siglas en inglés). Este modelo permite simular el efecto de la penetración de la generación fotovoltaica a pequeña escala en el sistema, validando las condiciones técnicas que debe garantizar el operador del sistema de distribución y calculando los costos de distribución, tanto los de capital como los de operación del sistema. Esta metodología puede ser aplicada bajo otros escenarios de penetración de GDF u otro tipo de generación.

\section{OTROS ANTECEDENTES}

La evolución de los sistemas de distribución ha estado marcada por cambios tecnológicos que le han permitido incrementar su viabilidad, tanto técnica como económica. La GD, contrario a lo que se puede pensar, es un concepto bajo el cual se desarrollaron los sistemas de distribución de energía eléctrica en sus inicios. Para ese entonces los sistemas eléctricos se encontraban compuestos por unidades de generación de pequeña escala ubicados cerca de los centros de carga, estos sistemas fueron inicialmente desarrollados en corriente directa. Con el tiempo, se fueron desarrollando grandes centrales de generación, con el objetivo de buscar economías de escala para el suministro de energía eléctrica. Fue de esta forma como se creó la necesidad de los sistemas de transmisión de energía que existen en la actualidad, con el objetivo principal de llevar la energía generada, por grandes unidades de generación, hasta los centros de demanda como ciudades y poblados.

En la actualidad, se observa como el sistema eléctrico está retornando a sus inicios en términos de localizar unidades de producción de energía a pequeña escala cerca de los centros de carga, e incluso, creando la posibilidad de independizar porciones del sistema dado que se puede contar con la energía suficiente para atender las necesidades energéticas de pequeños grupos de clientes, mediante generación a pequeña escala (Pepermans et al., 2015). El ingreso paulatino de generación a pequeña escala, cercana e incluso dentro de los centros poblados, se ha convertido en uno de los principales desafíos tanto para los distribuidores como para los entes reguladores. El diseño de mecanismos o marcos regulatorios adaptables a las nuevas condiciones tecnológicas e incentivar el uso de nuevas tecnologías de generación limpia, dados sus beneficios ambientales, son algunos de los desafíos para el regulador del mercado eléctrico. Por su parte, los operadores de redes de distribución deben, constantemente, evaluar los riesgos económicos y técnicos generados por el ingreso de las nuevas tecnologías al sistema. Las principales preocupaciones de los operadores incluyen: la confiabilidad del sistema, la calidad del servicio y la potencia, y obviamente la viabilidad económica del sistema que administran.

Colombia no ha sido ajena frente los cambios tecnológicos ni de penetración de nuevas tecnologías. No obstante, por sus características topológicas, su localización geográfica, y su riqueza hidrológica, Colombia ha desarrollado principalmente grandes plantas de generación hidroeléctrica. No obstante, de manera complementaria, su matriz energética posee algunas centrales de generación térmica. Existen algunos lugares del país que no pueden ser conectados a la red de transmisión nacional (por problemas de viabilidad económica de la infraestructura necesaria) y que son denominados zonas no interconectadas (ZNI). Es así como la penetración de GD en Colombia se encuentra en un estado incipiente, dado que aprovechando el potencial hídrico del país se ha explotado principalmente la generación de energía basado en grandes hidroeléctrica y, en otros casos, se han aprovechado las fuentes de agua que permiten la construcción de pequeñas plantas de generación. No obstante, dados los avances tecnológicos y la localización de Colombia en el mundo, esta presenta también un alto potencial de generación fotovoltaica, tanto de centros de generación a media escala en algunas regiones, como de pequeña escala en las ciudades.

Adicionalmente, el gobierno colombiano, por medio de su Ministerio de Minas y Energía y el regulador del mercado eléctrico, emitió la Ley 1715 en el año 2014, la cual tiene por objeto primordial regular la integración de las energías renovables no convencionales al sistema energético nacional. Esta Ley se convirtió en una clara señal de incentivo para los agentes del sector que deseen invertir en la generación a pequeña escala. Esto se debe, primordialmente, a que la Ley 1715 abre la posibilidad de que aquellos agentes, o usuarios, que generen más energía de la que se consumen puedan vender sus excedentes al sistema. Adicionalmente, incentiva la inversión en activos de generación a pequeña escala, considerando que los activos que se adquieran para este uso puedan ser depreciados con mayor rapidez que los convencionales y liberarlos de algunas cargas tributarias viabilizando financieramente este tipo de proyectos. Esta situación llama la atención a inversionistas del sector, considerando que las inversiones que se realicen tendrán un retorno más rápido. 
Las condiciones que se han citado, crean un entorno de alta incertidumbre para los operadores de red, que para el mercado colombiano son los distribuidores de energía, considerando que se han creado las condiciones necesarias para estimular la penetración de GD y esta situación tiene impactos sobre el sistema, los costos de operación y de capital asociados al sistema de distribución. Es así como, los operadores de red $(\mathrm{OR})$ se ven en la necesidad de estimar e identificar los riesgos e impacto de la penetración de GD en el sistema y, por su parte, el regulador requiere de herramientas que le permitan estimar el efecto en los costos de distribución de energía debidos a la GD. En el presente trabajo se estima el impacto sobre los costos de distribución considerando la penetración de GD, particularmente la fotovoltaica.

Los costos de distribución de energía eléctrica se representan mediante el TOTEX o gastos totales. Este a su vez, se divide en dos componentes: los costos de capital o CAPEX y los costos de operación; también conocidos como OPEX. En este caso, el CAPEX corresponde el reconocimiento de los activos productivos necesarios para la distribución de energía, mientras el OPEX representa los recursos asociados a las actividades de administrar, operar y mantener el sistema, entre otros. Adicionalmente, el OPEX considera los costos por el ingreso de nuevas tecnologías al sistema, como la GD (Gómez, 2013). Diferentes metodologías se emplean para la estimación de los costos que componen el costo total de la distribución. Dependiendo del esquema regulatorio bajo el cual operan los distribuidores, los costos de distribución pueden determinarse por medio de metodologías de Frontier Benchmarking o Norm Models. La estimación de costos mediante Frontier Benchmarking se efectúa empleando técnicas econométricas como las fronteras de eficiencia estocástica (SFA por sus siglas en inglés) o de programación lineal como el análisis envolvente de datos (DEA por sus siglas en inglés). Por su parte, los Norm Models son modelos de optimización o simulación basados en la representación ingenieril de los sistemas de distribución. Dentro de los Norm Models se encuentran los modelos de red de referencia (RNM por sus siglas en inglés) (Cossent, 2013). Estos últimos tienen la fortaleza de simular las condiciones técnicas cambiantes bajo las cuales operan los sistemas de distribución, estimando los costos operativos y las inversiones que se requieren para la prestación del servicio, situación que se presenta ante la penetración de GD. Esta característica hace de los RNMs una técnica adecuada para estimar los costos de distribución bajo la penetración de GD (Cossent et al., 2011).

Una de las tecnologías de generación a pequeña escala que más desarrollo ha logrado es la fotovoltaica. Por esta razón y ante las estimaciones de penetración de generación fotovoltaica realizadas en Colombia, es importante, tanto para los distribuidores como para el regulador, poseer una metodología que permita estimar el impacto en los costos de la distribución bajo la penetración de GDF. En el presente trabajo se emplea un modelo de red de referencia para la estimación de costos de distribución bajo la penetración de GDF. Los detalles de esta metodología se describen a continuación.

\section{METODOLOGÍA}

Cualquiera que sea la tecnología para producir energía a pequeña escala que sea empleada en GD, esta tiene consecuencias en las condiciones operativas y económicas del sistema de distribución. Desde el punto de vista operativo, la GD en ocasiones produce efectos positivos en términos de reducción de pérdidas y mejoras en la continuidad del servicio prestado (Gómez et al., 2018). Mientras, desde otro ángulo, pueden presentarse efectos adversos, todo esto dependiente de las condiciones particulares de cada caso (Pepermans et al., 2015). En términos económicos, los sistemas de distribución no responden en igual medida a la penetración de la GD. Esto se debe a que existe una alta dependencia entre el efecto de la penetración de GD relacionada con las características topológicas, constructivas y eléctricas de la infraestructura del sistema de distribución. Las condiciones citadas, confluyen y se convierten en efectos económicos que afectan directamente el costo de distribución, bien sea convertidos en costos operativos o en inversiones necesarias para garantizar la calidad del servicio prestado, incluso, porque el sistema puede requerir de refuerzos que garanticen su estabilidad y correcta operación. Dadas estas condiciones, la estimación de los costos de distribución bajo penetración de GD no puede evaluarse mediante metodologías que empleen costos históricos, puesto que puede incurrirse en imprecisiones, debido a que estas metodologías no capturan los efectos de los cambios tecnológicos en la infraestructura. Por esta razón, la estimación de costos de distribución bajo penetración de GD debe realizarse mediante herramientas de optimización que permitan simular las condiciones técnicas cambiantes del sistema y que puedan garantizar la correcta operación del sistema, bajo el nivel de costos adecuado.

En la literatura técnica se han desarrollado metodologías que permiten comparar las empresas de distribución frente a un sistema o red de referencia diseñado mediante criterios óptimos. Estos son los denominados Norm Models o RNMs (Cossent, 2013). La aplicación de los RNMs con fines comparativos es de utilidad en el desarrollo de marcos regulatorios o la definición de la remuneración de empresas de distribución. No obstante, estos modelos también pueden emplearse para la planeación, dada su capacidad de simular el desempeño técnico de las redes que componen el sistema de distribución. 
Los RNMs son modelos de optimización de las redes de distribución que permiten construir redes de referencia que pueden ser usadas tanto para la planeación de las empresas como para la estimación de costos óptimos del distribuidor, obteniendo tanto el CAPEX como el OPEX (Domingo et al., 2011; GarcíaMontoya et al., 2020). Para su aplicación se ingresa al modelo información detallada sobre la localización de las cargas o clientes, los costos de mantenimiento, características eléctricas de la infraestructura, subestaciones existentes y/o disponibles, como también objetivos o metas de indicadores de calidad del servicio, según sea la aplicación. Con esta información, el RNM diseña la red óptima necesaria para la atención de los clientes, cumpliendo con las exigencias técnicas y operativas requeridas para una adecuada prestación del servicio. Los RNMs pueden ejecutarse bajo dos enfoques, un enfoque desde cero o modo greenfield y un modelo de planeación de expansión o modo brownfield. El modelo greenfield, bajo un escenario genérico, partiendo de la localización de las cargas georeferenciadas y la ubicación de unas subestaciones de distribución, construye las redes necesarias para la prestación del servicio, siempre en búsqueda de la red óptima bajo criterios mínimos de calidad del servicio y de pérdidas del sistema (GarcíaMontoya y Lopez-lezama, 2016).

Por otro lado, el modo de expansión o brownfield, se parte de una infraestructura existente y, según es escenario que se desea simular, se le suministra información al modelo de modo tal que él diseñe las redes óptimas requeridas para la prestación del servicio, pero en este caso bajo unas condiciones de servicio diferentes a las originales. La salida del modelo es un sistema de distribución óptimo con un informe de las modificaciones requeridas por el sistema inicial para cumplir con las necesidades que se le demandaron en el escenario propuesto, cuantificando, adicionalmente, los costos asociados al sistema original y las nuevas necesidades. En ambos modos, greenfield y brownfield, el RNM efectúa una estimación de los indicadores de calidad del servicio esperados y las pérdidas técnicas del sistema de distribución, con lo cual se puede validar, adicionalmente, cual es el nivel de pérdidas y de calidad del servicio esperados. Mayores detalles sobre los modelos de red de referencia pueden ser consultados en (Cossent, 2013).

En el presente trabajo se empleó el RNM en sus dos modos, greenfield y brownfield. Inicialmente se suministra la localización de las cargas existentes, además de la información técnico económica básica necesaria, y se le permite al modelo crear, bajo el modo greenfield, las redes necesarias para la atención del servicio, limitando al modelo la posibilidad de crear o localizar nuevas subestaciones. Con esta restricción se logra que la topología de la red sea similar al tipo de construcción en el mercado Colombiano, el cual es predominantemente radial. Luego, empleando el RNM en modo brownfield, se evalúan diversos escenarios de penetración de GDF. Para esto se ingresan como redes existentes la red de referencia obtenida al ejecutar el modo greenfield. El objetivo primordial de esta simulación es poder identificar el efecto que pueda tener la penetración de la GDF sobre los costos de distribución, garantizando los límites operativos que se le demandan en términos de calidad del servicio. Una vez ejecutadas ambas, se procede al análisis de la información obtenida de las diferentes simulaciones.

Empleando el modelo de red de referencia, en el presente trabajo se efectuaron diferentes simulaciones de la penetración de GDF en un caso de estudio construido con infraestructura típica colombiana. El caso de estudio está conformado por una muestra de 1000 transformadores de distribución, que atienden a 11000 clientes aproximadamente. De acuerdo con proyecciones para Colombia se efectuaron simulaciones de penetración de GDF entre el 0 y el $32 \%$. Este porcentaje se calcula respecto a la potencia instalada total del sistema. Detalles mayores sobre las simulaciones y el caso de estudio se analizan en la sección resultados.

\section{RESULTADOS}

Para estimar el impacto de la penetración de la GDF sobre los costos de distribución bajo las condiciones de la infraestructura típica colombiana se empleó un sistema de distribución de 1000 transformadores de distribución. Los transformadores elegidos pertenecen a una porción de un sistema de distribución real el cual atiende un número aproximado de 11000 clientes. Como característica topológica principal los clientes se encuentran emplazados en una región que se caracteriza entre rural y urbana, dado que algunos de ellos se encuentran en pequeños centros poblados o en cabeceras municipales, como se les denomina en Colombia. En la Tabla 1 pueden apreciarse las características técnicas del caso de estudio.

Tabla 1: características técnicas del caso de estudio empleado

\begin{tabular}{|l|l|}
\hline Capacidad instalada inicial & 9447 [KW] \\
\hline Capacidad de los transformadores de distribución & Min: 10, Max: 75 [kVA] \\
\hline Niveles de tensión & $13.2,44[\mathrm{kV}]$ \\
\hline Rango de cargas & Min: 3, Max: 30 [kVA] \\
\hline Demanda esperada anual & 41.9 [GWh/año] \\
\hline
\end{tabular}


Para la ejecución del RNM en el caso de estudio seleccionado, se suministró al modelo la información de la localización de cargas y transformadores de distribución, al igual que los limites esperados de calidad del servicio para una región rural y urbana, dado que el caso de estudio considera ambos casos en forma simultánea. Adicionalmente, se ingresó el costo de la infraestructura reconocida por el regulador por unidades constructivas, de modo tal que la red que diseñe el modelo sea construida bajo los costos de inversión reconocidos por el regulador y, por otra parte, se ingresan los costos por hora hombre para el mantenimiento de las redes. Por último, se ingresaron datos relacionados con las tasas de falla de equipos, con esto el modelo estima los indicadores de calidad del servicio esperados. Con la información recopilada se ejecuta el modelo RNM greenfield. Una vez se obtiene el resultado de la red inicial, este resultado es empleado como insumo del RNM brownfield, mediante el cual se simula la penetración de GDF.

Para la simulación de la penetración de GDF se eligieron unidades a pequeña escala, con una capacidad de

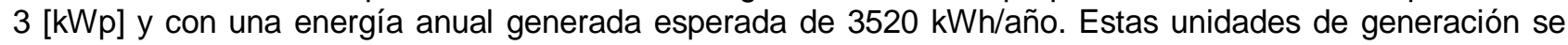
eligieron en consonancia con los estudios realizados por la UPME, quien dentro de su rol de planeación energética nacional, ha desarrollado estudios en los que se estimaron los costos indicativos de diferentes tipos de generación en la economía Colombiana y los diferentes tipos de tecnologías de generación de energía con probabilidad de penetrar en el mercado colombiano. La UPME en su estudio sobre Integración de las energías renovables no convencionales en Colombia, realizó un análisis del potencial y proyecciones para el aprovechamiento de energía solar a través de sistemas solar FV en techos urbanos (UPME, 2015). Los resultados de este estudio muestran los niveles esperados de penetración bajo de GDF bajo las condiciones mencionadas, la cual es usada como referente en el presente trabajo. La proyección de la GDF es simulada colocando de manera aleatoria generadores fotovoltaicos en instalaciones ya existentes, teniendo en cuenta como magnitud base para la penetración el monto potencial estimado por el estudio ya mencionado. Con esto se simula el escenario bajo el cual un cliente del sistema adquiere e instala una unidad de generación a pequeña escala, con el objetivo de autoabastecerse y, eventualmente como lo contempla la ley, poder inyectar la energía remanente al sistema.

Con el objetivo de efectuar la simulación de la penetración de GDF ajustada a la realidad colombiana, se emplean las curvas de demanda típica y la curva de generación típica de los paneles. Esta última dependiendo de la radiación solar en Colombia. Es importante notar que si las simulaciones se realizan considerando solamente el momento de generación plena (100\%) de los paneles fotovoltaicos, solo se estaría evaluando ese momento del día, pero en la realidad puede requerirse de la evaluación del total de la curva de modo tal que se identifique el potencial de energía diario que puede autogenerarse en el sistema, proveniente de los paneles. Las curvas típicas empleadas en el caso de estudio, expresadas en porcentaje, pueden apreciarse en la Fig. 1.

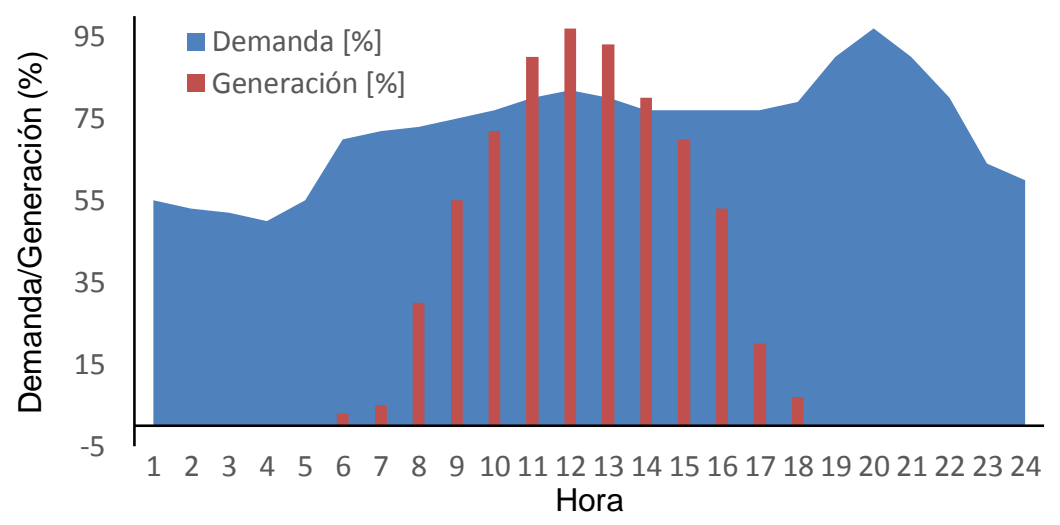

Fig. 1: Curva de demanda típica y curva de generación fotovoltaica característica.

Bajo las condiciones descritas anteriormente se ejecutaron diferentes simulaciones sobre el caso de estudio. Una vez se obtuvo la red inicial con el RNM greenfield, se ejecutó la simulación de la penetración mediante el RNM brownfield. La GDF fue localizada de manera aleatoria en las cargas ya existentes. Este proceso de simulación se realizó iniciando en 50 unidades de GDF hasta llegar a 1000 de ellas. Para cada una de ellas se recopiló la información de costos de operación y de infraestructura, los kilómetros de red del sistema y los indicadores de calidad esperados según la simulación, entre otros detalles. Esta información se presenta en la Tabla 2. Note que se realizaron simulaciones que llegan a una participación de GDF del $31.8 \%$ respecto a la capacidad instalada, la cual corresponde a una penetración significativa, si se visualiza desde la expectativa de penetración nacional que estima la UPME para los siguientes 10 años (UPME, 2015). Con esto se logra simular en el caso de estudio el efecto de la penetración de GDF bajo condiciones superiores a las esperadas 
por esa entidad y tener señales relacionadas con el efecto de esta en los costos en los que incurren los OR. De esta información se obtuvo que principalmente los valores de costos de inversión de la infraestructura están estimados en 39033 [MCOP - Millones de pesos colombianos] y los costos de mantenimiento en 3020 [MCOP]. Adicionalmente, no se observan mayores cambios en la longitud de la red. Esto significa que las características técnicas de la infraestructura colombiana no demandan mayores modificaciones en las redes de distribución para la atención de la penetración de la GDF, obviamente, bajo las condiciones específicas en las que se realizaron las simulaciones.

Tabla 2: Resultados obtenidos en las simulaciones de penetración de GDF.

\begin{tabular}{|l|l|l|l|l|l|l|l|}
\hline \multicolumn{2}{|c|}{ Cálculos de GDF conectada en media tensión } & \multicolumn{2}{|c|}{ Cálculo de penetración de GDF } & \multicolumn{2}{c|}{ Infraestructura } \\
\hline Simulación & SAIDI & SAIFI & $\begin{array}{c}\text { Potencia } \\
\text { GDF }[\mathrm{kW}]\end{array}$ & $\begin{array}{c}\text { Según Potencia } \\
\text { Instalada }\end{array}$ & $\begin{array}{c}\text { Según Número } \\
\text { de Clientes }\end{array}$ & $\begin{array}{c}\text { Capacidad } \\
\text { incluye GDF } \\
{[M V A]}\end{array}$ & $\begin{array}{c}\text { Kilómetros } \\
\text { de red }\end{array}$ \\
\hline GDF 50 & 33.17 & 9.34 & 150 & $1.6 \%$ & $0.5 \%$ & 9.597 & 660.46 \\
\hline GDF 100 & 33.27 & 9.35 & 300 & $3.2 \%$ & $1.0 \%$ & 9.747 & 660.46 \\
\hline GDF 150 & 33.2 & 9.38 & 450 & $4.8 \%$ & $1.4 \%$ & 9.897 & 660.46 \\
\hline GDF 200 & 32.93 & 9.32 & 600 & $6.4 \%$ & $1.9 \%$ & 10.047 & 660.46 \\
\hline GDF 250 & 32.51 & 9.21 & 750 & $7.9 \%$ & $2.4 \%$ & 10.197 & 660.46 \\
\hline GDF 500 & 32.03 & 9.11 & 1500 & $15.9 \%$ & $4.8 \%$ & 10.947 & 660.46 \\
\hline GDF 1000 & 33.94 & 9.63 & 3000 & $31.8 \%$ & $9.5 \%$ & 12.447 & 660.46 \\
\hline
\end{tabular}

Algunos trabajos similares al desarrollado en este artículo han sido realizados a nivel internacional. Estos sirven de referente para la simulación ejecutada. En (Cossent et al., 2011), se presenta un análisis de los costos de la distribución bajo penetración de GD en tres países diferentes de Europa. En este trabajo se observan los efectos de la penetración de GD en los Países Bajos, Alemania y España. El desarrollo de ese trabajo considera, además de la penetración de GD, el crecimiento de la demanda. Dentro de los resultados más relevantes de ese trabajo se encuentra que, si bien en general la penetración de GD demanda mayores costos de la distribución, no todas lo demandan debido a características técnicas del sistema de distribución. Un caso similar sucede con los resultados obtenidos en el presente estudio, apreciándose como no se identifica la necesidad de grandes inversiones en la infraestructura de las redes de distribución frente a la penetración de GDF. Por otra parte, no se visualizan efectos mayores sobre la calidad del servicio prestado dado que los indicadores de calidad SAIDI y SAIFI no se afectaron en mayor medida con el ingreso de la GD.

\section{DISCUSIÓN}

La GDF a nivel mundial se visualiza como una de las fuentes alternativas de generación renovable que más desarrollo ha logrado en los últimos años. Para los distribuidores de energía eléctrica la apropiada inclusión de este tipo de GD en sus redes representa nuevos desafíos. Los OR colombianos no están al margen de esta situación, dado que se espera una creciente participación de GDF en el corto y mediano plazo, debido especialmente a los incentivos desarrollados por el gobierno nacional. Considerando las anteriores condiciones, en el presente trabajo se desarrolló un estudio que permite estimar el efecto sobre los costos de la distribución de energía, debidos a la penetración de la GDF bajo el contexto constructivo y las proyecciones de penetración de Colombia. Vale la pena anotar que si se desea extrapolar estos resultados a otro entorno, es necesario revisar los supuestos, las proyecciones y condiciones particulares de otros entornos o situaciones, particularmente porque las características constructivas del sistema de distribución inciden considerablemente y las características topológicas presentes en el caso de estudio inciden sobre las necesidades de inversión que se puedan derivar de la instalación de los paneles fotovoltaicos, especialmente por los cambios en los flujos de energía en el sistema que se derivan en refuerzos del mismo y las consecuentes inversiones.

Como pudo apreciarse en los resultados obtenidos, la expectativa de penetración de GDF para el caso colombiano no presenta una alta incidencia dentro de los costos del OR. Esto se debe, inicialmente, a que no se espera en el corto plazo una penetración alta de GDF. Por otra parte, dada la tasa de crecimiento esperada de la demanda en Colombia, estando cerca de un $2 \%$ anual, puede permitir que los refuerzos necesarios para la atención de la penetración de GDF a pequeña escala se anulen con las inversiones necesarias por el crecimiento de la demanda. Adicionalmente, las condiciones topológicas de las redes de distribución colombianas, como lo es su predominante radialidad, hacen que las redes se construyan para cumplir con las caídas de tensión mínimas requeridas, convirtiéndolas en redes con conductores robustos que pueden atender una mayor demanda y no son especialmente sensibles a la GDF a pequeña escala. Por último, pese a que no se avizora un impacto en costos de distribución en el corto plazo bajo magnitudes pequeñas de penetración, este problema debe continuarse investigando de modo tal que se tenga elementos suficientes para prever el efecto de la penetración de la GD mediante otras tecnologías de generación y valores mayores de GDF, minimizando la incertidumbre y su impacto final. 


\section{CONCLUSIONES}

En este artículo se presentó una metodología para determinar el costo de distribución de la energía eléctrica en Colombia considerando la integración de generación distribuida fotovoltaica. La evaluación del impacto de la generación distribuida fotovoltaica se llevó a cabo mediante modelos de red de referencia. Se realizaron pruebas en un sistema real con 1000 transformadores de distribución y 11000 clientes. De acuerdo a los resultados obtenidos, se pueden plantear las siguientes conclusiones: (i) Bajo el entorno, planteamientos y supuestos planteados en la metodología utilizada, se concluye que al nivel de penetración de GDF (bajo las proyecciones esperadas segun estudios de referencia) los costos del distribuidor (CAPEX+OPEX) no presentan efectos considerables; (ii) Los indicadores de calidad esperada no se ven afectados por la penetración de GDF, esto se debe a que no se considera la inclusión de almacenamiento de energía de manera conjunta con la generación fotovoltaica; (iii) Los resultados obtenidos muestran que dadas las caracteristicas técnicas y construtivas del sistema hacen que no se requieran refuerzos mayores que conyeven inversiones que afecten los costos del distribuidor. Es importante tener en cuenta que dado que los sistemas fotovoltaicos propuestos son de pequeña escala, el sistema no requeriría ajustes importantes en materia de protecciones, lo cual si se dar'pia en caso de que estos tuvieran una escala mediana o grande; y (iv) El presente estudio fue desarrollado bajo condiciones específicas de penetración, expectativas de crecimiento, características técnicas del sistema de distribución y condiciones particulares para operadores de red en Colombia, las cuales permiten concluir que la metodología empleade empleando el modelo de red de referencia es adecuada, pero en caso de desearse extrapolar la metodología para un entorno diferente se requiere revisar las condiciones y supuestos utilizados.

\section{REFERENCIAS}

Adefarati, T., y Bansal, R.C. Integration of renewable distributed generators into the distribution system: a review, doi.org/10.1049/iet-rpg.2015.0378, IET Renewable Power Generation 7(2), 873-884 (2016)

Branker, K., Pathak, M.J.M., y Pearce, J.M., A review of solar photovoltaic levelized cost of electricity, doi.org/10.1016/j.rser.2011.07.104, Renewable and Sustainable Energy Reviews, 15(9), 4470-4482 (2011).

Cossent, R., Olmos, L., Gómez, T., Mateo, C., y Frías, P., Distribution network costs under different penetration levels of distributed generation., doi.org/10.1002/etep.503, European Transactions on Electrical Power, 21(6), 1869-1888 (2011)

Cossent, R., Economic regulation of distribution system operators and its adaptation to the penetration of distributed energy resources and smart grid technologies, Universidad Pontificia Comillas, Madrid, España (2013)

Domingo, C.M., Roman, T.G.S. y otros tres autores, A Reference Network Model for Large-Scale Distribution Planning With Automatic Street Map Generation, doi.org/10.1109/TPWRS.2010.2052077, IEEE Transactions on Power Systems, 26(1), 190-197 (2011)

Eltawil, M.A., y Zhao, Z., Grid-connected photovoltaic power systems: Technical and potential problems - a review, doi.org/10.1016/j.rser.2009.07.015, Renewable and Sustainable Energy Reviews, 14(1), 112-129 (2010).

Gallego J.D., Franco, C.J., y Zapata, S., Policiies for the utilization of hydropower potential in Colombia using small plants, doi.org/10.1109/TLA.2015.7404918, IEEE Latin America Transactions, 13(12), 2844-3850 (2015).

García-Montoya, C.A. y J.M. López-Lezama., Caracterización del costo de distribución de energía eléctrica mediante modelos de frontera de eficiencia considerando un indicador de calidad del servicio, doi.org/10.4067/S071807642017000200005, Revista Información Tecnológica, 28(2), 37-46, (2016)

García-Montoya, C.A., López-Lezama. J.M. y San Román T.G., Estimación del gasto total (TOTEX) de empresas de distribución mediante modelos de red de referencia y de fronteras de eficiencia, dx.doi.org/10.4067/S071807642020000100183, Revista Información Tecnológica, 31(1), 183-192 (2020)

Gómez, T., Monopoly Regulation; in Regulation of the Power Sector by I. Pérez Arriaga pp 151-198. Springer, London, United Kingdom (2013)

Gómez, V.A., Hernández, C. y Rivas, E., La influencia de los niveles de penetración de la generación distribuida en los mercados energéticos, dx.doi.org/10.4067/S0718-07642018000100117, Revista Información Tecnológica, 29(1), 117-128 (2018)

Hassan, M.H., Abdullah, M.A.S., Ali, M.E. y Abdullrahman, A., A novel crow search algorithm auto-drive PSO for optimal allocation and sizing of renewable distributed generation, doi.org/ 10.1109/ACCESS.2020.2968462, IEEE Access, 8(1), 27807-27820 (2020)

Huang L., Chen, Z., y otros cuatro autores, Optimal planning of renewable energy source and anergy storage in a medium and low-voltage distributed AC/DC system in China, doi.org/1010.1049/joe.2018.8546, The Journal of Engineering, 16(3), 2354-2361 (2019)

Kerekes, T., Koutroulis, E., y otros tres autores, An optimization method for designing large PV plants, doi.org/10.1109/JPHOTOV.2012.2230684, IEEE Journal of Photovoltaics, 3(2), 814-822 (2013)

Moore, L.M., y Post, H.N., Five years of operating experience at a large, utility-scale photovoltaic generating plant. doi.org/10.1002/pip.800, Progress in Photovoltaics: Research and Applications, 16(3), 249-259 (2008) 
Normandia L,F., Salles, M.B.C., y Machado R., Technical cost of operating a photovoltaic installation as a STATCOM at nighttime, doi.org/10.1109/TSTE.2018.2825104, IEEE Transactions on Sustainable Energy, 10(1), 75-81 (2019)

Paul, A.B., Understanding manufacturing cost influence on future trends in silicon photovoltaics, doi.org/10.1109/JPHOTOV.2014.2358081, IEEE Journal of Photovoltaics, 4(6), 1477-1482 (2014)

Pepermans, G., Driesen, J., Haeseldonckx, D., Belmans, R., \& D’haeseleer, W., Distributed generation: Definition, benefits and issues, doi.org/10.1016/j.enpol.2003.10.004, Energy Policy, 33(6), 787-798 (2005)

Pereira R.B., Martis, G.R., Contreras, J., y Mantovani, J.R.S., Optimal distribued generation and reactive power allocation in electrical distribution systems, doi.org/10.1109/TSTE.2015.2512819, IEEE Transactions on Sustainable Energy, 7(3), 795-984 (2016)

Restrepo A.R., Manotas, D.F., y Lozano C.A., Self-generation of electricity, assessment and optimization under the new support schemes in Colombia, doi.org/10.1109/TLA.2016.7459614, IEEE Latin America Transactions, 14(3), 1308-1314 (2016)

Toledo, O.M., Oliveira Filho, D., y Diniz, A.S.A.C., Distributed photovoltaic generation and energy storage systems: A review, doi.org/10.1016/j.rser.2009.08.007, Renewable and Sustainable Energy Reviews, 14(1), 506-511 (2010).

UPME, Integración de las energías renovables no convencionales en Colombia, Bogotá, Colombia, (2015). Estudio disponible online: http://www.upme.gov.co/Estudios/2015/Integracion_Energias_Renovables.

Vasileios, A.E., y Pavlos S.G., Optimal distribued generation placement under uncertainties based on point estimate method embedded genetic algorithm, doi.org/10.1049/iet-gtd.2013.0442, IET Generation Transmision and Distribution, 8(3), 389-400 (2014).

Wang, P., Wang, W., y Xu Dianguo, Optimal sizing of distributed generation in DC microgrids with comprehensive consideration of sytem operation modes and operation targets, doi.org/10.1109/ACCESS.2018.2842119, IEEE Access, 6(1), 31129-31140 (2018)

Yong C., Ji, D., Wen, L., Yunlong, C. y Li, C., Research on the cost of distributed photovoltaic plant of China based on whole life cycle perspective, doi.org/10.1109/ACCESS.2019.2924318, IEEE Acesss, 7(1), 89379-89389 (2019)

Zhan, H., Wang, C., y otros cinco autores, Relay protection coordination integrated optimal placement and sizing of distributed generation sources in distribution networks, doi.org/10.1109/TSG.2015.2420667, IEEE Transactions on Smart Grids, 7(1), 55-65 (2016)

Zou, B., Wang, J., y Wen, F., Optimal investment strategies for distributed generation in distribution networks with real option analysis, doi.org/10.1049/iet-gtd.2016.0541, IET Generation Transmission and Distribution, 11(3), 804-813 (2017) 
